\title{
PRECISE SOLUTION OF LAPLACE'S EQUATION
}

\author{
ZHIXIN SHI AND BRIAN HASSARD
}

\begin{abstract}
An approximate method is described for solving Laplace's equation

$$
\left\{\begin{aligned}
\Delta u=0 & \text { in } \Omega=(0,1) \times(0,1), \\
\left.u\right|_{\partial \Omega}=g & \text { on } \partial \Omega
\end{aligned}\right.
$$

precisely in the sense of Aberth's 1988 monograph. The algorithm uses singularity extraction, Fourier series methods, Taylor series methods, and interval analysis to construct an approximation $U(x, y)$ to the solution $u(x, y)$ at points in the square, and a uniform bound on the error $|U(x, y)-u(x, y)|$. The algorithm applies to problems in which the boundary data $g$ is specified in terms of four elementary functions. The boundary data may be discontinuous at the corners.
\end{abstract}

\section{INTRODUCTION}

Consider Laplace's equation

$$
\left\{\begin{aligned}
\Delta u=0 & \text { in } \Omega, \\
\left.u\right|_{\partial \Omega}=g & \text { on } \partial \Omega,
\end{aligned}\right.
$$

where $\Omega$ is the unit square $(0,1) \times(0,1)$ and $\partial \Omega$ is the boundary of $\Omega$. We suppose that $g$ is defined on the sides $\partial \Omega_{j}, j=1,2,3,4$, of the square by continuous elementary functions $g_{1}(x), g_{2}(y), g_{3}(x)$, and $g_{4}(y)$. Here, $\partial \Omega_{j}, j=1,2,3,4$, are labeled counterclockwise starting with $\partial \Omega_{1}=\{0 \leq$ $x<1, y=0\}$.

For given positive integers $n, N$, the algorithm we describe determines an approximate solution $U=U_{n}, N_{N}$ and an $\epsilon=\epsilon_{n, N}$ such that $\mid u(x, y)-$ $U(x, y) \mid<\epsilon$ uniformly for $(x, y) \in \Omega$. Also, $\epsilon_{n, N}=\epsilon_{n, N}^{\text {round }}+\epsilon_{n, N}^{\text {trunc }}$, where $\epsilon_{n, N}^{\text {round }}$ may be made arbitrarily small by increasing the precision of the interval arithmetic, and for each fixed $n, \epsilon_{n, N}^{\text {trunc }}=O\left(N^{-2 n-1}\right), N \rightarrow \infty$.

There is an enormous body of literature on numerical methods for linear elliptic partial differential equations, and a variety of packages available, such as [5]. There are methods capable of high-accuracy solution [3], methods for which $H^{1}$ error bounds [8] and methods for which pointwise error bounds [2] have been derived. The present work combines a high-accuracy scheme with a pointwise bounding argument and methods of interval analysis to completely control

Received by the editor September 27, 1993 and, in revised form, February 28, 1994.

1991 Mathematics Subject Classification. Primary 35J05, 35J25, 35A40, 65N15; Secondary 65T10, 42A16, 65D30. 
both truncation and roundoff error. The precision of the results is guaranteed. We believe that our scheme is the only one presently implemented which can make this claim. Our application of interval analysis uses precise methods described by Aberth [1] for linear algebra, differentiation, and quadrature. Our scheme is potentially useful for testing the accuracy of other schemes for solving general linear elliptic partial differential equations.

The main idea in our scheme for (1.1) can be considered as a method for accelerating the convergence of Fourier series solutions. The convergence rate of such a series is limited by nonzero values of even-order derivatives of the boundary data $g_{j}, j=1,2,3,4$, at the corners. As the functions $g_{j}, j=$ $1, \ldots, 4$, are elementary, their derivatives up to any fixed order at the corners are computable (i.e., can be computed precisely, cf. Aberth [1, Chapter 3]). We first use the known derivatives of the boundary data at the corners to convert (1.1) to a problem of the same form, but for which the even derivatives of the boundary data at the corners vanish up to a certain order.

The resulting Laplace's equation is solved using a truncated Fourier series method. The truncation error is bounded using uniform bounds for $2 k$ thorder derivatives of the boundary data, which are again computable. Taylor series methods are combined with interval analysis to obtain these bounds; the approach is similar to that used by Aberth [1, Chapter 3] in precise methods for quadrature.

We call part of the scheme for (1.1) "singularity extraction", since singularities of partial derivatives of the solution at the corners are removed.

It is well known that the solution $u$ of (1.1) is the sum of the solutions $u_{j}, j=1, \ldots, 4$, of the problems

$$
\left\{\begin{array}{l}
\Delta u_{j}=0 \\
\left.u_{j}\right|_{\partial \Omega_{j}=g_{j},} \quad \text { in } \Omega, \\
\left.u_{j}\right|_{\partial \Omega_{k}}=0, \quad k \neq j,
\end{array}\right.
$$

with nonzero data only on $\partial \Omega_{j}, j=1, \ldots, 4$. The solution of (1.2) with $j=1$ can be easily written as

$$
u_{1}(x, y)=\sum_{n=1}^{\infty} a_{n} \frac{\sinh n \pi(1-y)}{\sinh n \pi} \sin n \pi x,
$$

where $a_{n}=2 \int_{0}^{1} g_{1}(x) \sin n \pi x d x$, and there are similar expressions for the solutions $u_{j}, j=2,3,4$.

An approximate solution of (1.1) can be found by truncating the Fourier series (1.3) and the other three Fourier series. However, these Fourier series do not in general converge fast enough to be the basis of a high-accuracy scheme unless the boundary data has additional properties. This motivates the following definitions:

Definition 1.1 (Property $A_{k}$ ). For any integer $k \geq 0$, we say the boundary data $g$ has property $A_{k}$ if 


$$
\begin{gathered}
\lim _{x \rightarrow 0^{+}} g_{1}^{(2 j)}(x)=(-1)^{j} \lim _{y \rightarrow 0^{+}} g_{4}^{(2 j)}(y), \\
\lim _{x \rightarrow 1^{-}} g_{1}^{(2 j)}(x)=(-1)^{j} \lim _{y \rightarrow 0^{+}} g_{2}^{(2 j)}(y), \\
\lim _{y \rightarrow 1^{-}} g_{2}^{(2 j)}(y)=(-1)^{j} \lim _{x \rightarrow 1^{-}} g_{3}^{(2 j)}(x), \\
\lim _{y \rightarrow 1^{-}} g_{4}^{(2 j)}(y)=(-1)^{j} \lim _{x \rightarrow 0^{+}} g_{3}^{(2 j)}(x)
\end{gathered}
$$

for all $j=0, \ldots, k$, and all the limits exist. We say a problem (1.1) has property $A_{k}$ if the associated boundary data has property $A_{k}$.

Definition 1.2 (Property $b_{k}$ ). For $k \geq 0$, we say that the boundary data $g$ has property $b_{k}$ if

$$
\begin{gathered}
\lim _{x \rightarrow 0^{+}} g_{1}^{(2 k)}(x)=\lim _{y \rightarrow 0^{+}} g_{4}^{(2 k)}(y)=0, \\
\lim _{x \rightarrow 1^{-}} g_{1}^{(2 k)}(x)=\lim _{y \rightarrow 0^{+}} g_{2}^{(2 k)}(y)=0, \\
\lim _{y \rightarrow 1^{-}} g_{2}^{(2 k)}(y)=\lim _{x \rightarrow 1^{-}} g_{3}^{(2 k)}(x)=0, \\
\lim _{y \rightarrow 1^{-}} g_{4}^{(2 k)}(y)=\lim _{x \rightarrow 0^{+}} g_{3}^{(2 k)}(x)=0 .
\end{gathered}
$$

We say the problem (1.1) has property $b_{k}$ if the associated boundary data has property $b_{k}$.

Definition 1.3 (Property $B_{k}$ ). We say that the boundary data $g$ has property $B_{k}$ if it has property $b_{j}$ for $j=0,1, \ldots, k$, and we say the problem (1.1) has property $B_{k}$ if the associated boundary data has property $B_{k}$.

The basic idea of the scheme we describe, is to convert a general problem (1.1) which may not even have continuous boundary data (property $A_{0}$ ) to a problem of the same form (1.1) but with property $B_{n}$ for some given $n$. This is done by subtracting linear combinations of specific harmonic functions in two stages. The first stage takes the general problem (1.1) to one with property $A_{n}$, and may be called singularity elimination because the harmonic functions employed have corner singularities. The second stage takes a problem with property $A_{n}$ to one with property $B_{n}$ by subtracting a polynomial. The resulting problem is then solved by classical Fourier series methods: if a problem (1.1) has property $B_{n}$ for some sufficiently large $n$, then the Fourier series solution converges rapidly (see $\S 4)$.

\section{Conversion to a BVP with Property $A_{n}$}

In this section we shall convert the boundary value problem (1.1) to a problem of the same general form but with "better" boundary data, i.e., having property $A_{n}$ for some large $n$. It is not by itself sufficient for fast convergence of Fourier series solutions that a problem has property $A_{n}$ for some large $n$. However, property $A_{n}$ is necessary, and converting (1.1) is the first step. We have the following 
Lemma 2.1. (1) For any integer $k \geq 0$, the function

$$
V_{k}(x, y)=r^{2 k}(\cos (2 k \theta) \theta+\sin (2 k \theta) \ln r),
$$

where $x=r \cos \theta$ and $y=r \sin \theta$, is harmonic. Also the restriction $\left.V_{k}\right|_{\partial \Omega}$ has property $A_{k}$ at $(1,0),(1,1)$ and $(0,1)$, but not at $(0,0)$. Explicitly, for $j \geq 0$,

$$
\begin{aligned}
& \lim _{x \rightarrow 0^{+}} \frac{d^{2 j}}{d x^{2 j}} V_{k}(x, 0)=0, \\
& \lim _{y \rightarrow 0^{+}} \frac{d^{2 j}}{d y^{2 j}} V_{k}(0, y)=(-1)^{k} \frac{\pi}{2}(2 k) ! \delta_{j k}
\end{aligned}
$$

(2) A general problem (1.1) can be converted to a problem of the same form (1.1) which has property $A_{0}$ by subtracting a linear combination of the functions $V_{0}^{1}(x, y) \equiv V_{0}(x, y), V_{0}^{2}(x, y) \equiv V_{0}(y, 1-x), V_{0}^{3}(x, y) \equiv V_{0}(1-x, 1-y)$, and $V_{0}^{4}(x, y) \equiv V_{0}(1-y, x)$.

(3) A problem (1.1) which has property $A_{k-1}$ for some $k \geq 1$ can be converted to a problem of the same form (1.1) which has property $A_{k}$ by subtracting a linear combination of the functions $V_{k}^{1}(x, y) \equiv V_{k}(x, y), V_{k}^{2}(x, y) \equiv V_{k}(y, 1-x)$, $V_{k}^{3}(x, y) \equiv V_{k}(1-x, 1-y)$, and $V_{k}^{4}(x, y) \equiv V_{k}(1-y, x)$.

Proof. Assertion (1) is easily verified.

To prove (2), we set $u_{0}=u-\sum_{i=1}^{4} \alpha_{0 i} V_{0}^{i}$, where

$$
\begin{aligned}
& \alpha_{01}=\frac{2}{\pi}\left(\lim _{y \rightarrow 0^{+}} g_{4}(y)-\lim _{x \rightarrow 0^{+}} g_{1}(x)\right), \\
& \alpha_{02}=\frac{2}{\pi}\left(\lim _{x \rightarrow 1^{-}} g_{1}(x)-\lim _{y \rightarrow 0^{+}} g_{2}(y)\right), \\
& \alpha_{03}=\frac{2}{\pi}\left(\lim _{y \rightarrow 1^{-}} g_{2}(y)-\lim _{x \rightarrow 1^{-}} g_{3}(x)\right), \\
& \alpha_{04}=\frac{2}{\pi}\left(\lim _{x \rightarrow 0^{+}} g_{3}(x)-\lim _{y \rightarrow 1^{-}} g_{4}(y)\right) .
\end{aligned}
$$

Then $u_{0}$ satisfies

$$
\begin{cases}\Delta u_{0}=0 & \text { in } \Omega, \\ \left.u_{0}\right|_{\partial \Omega}=g-\left.\sum_{i=1}^{4} \alpha_{0 i} V_{0}^{i}\right|_{\partial \Omega} & \text { on } \partial \Omega,\end{cases}
$$

and the new boundary data has property $A_{0}$. 
To prove (3), we now suppose that problem (1.1) has property $A_{k-1}$ with $k \geq 1$. Set $u_{k}=u-\sum_{j=1}^{4} \alpha_{k j} V_{k}^{j}$, where

$$
\begin{aligned}
& \alpha_{k 1}=\frac{2}{\pi(2 k) !}\left((-1)^{k} \lim _{y \rightarrow 0^{+}} g_{4}^{(2 k)}(y)-\lim _{x \rightarrow 0^{+}} g_{1}^{(2 k)}(x)\right), \\
& \alpha_{k 2}=\frac{2}{\pi(2 k) !}\left((-1)^{k} \lim _{x \rightarrow 1^{-}} g_{1}^{(2 k)}(x)-\lim _{y \rightarrow 0^{+}} g_{2}^{(2 k)}(y)\right), \\
& \alpha_{k 3}=\frac{2}{\pi(2 k) !}\left((-1)^{k} \lim _{y \rightarrow 1^{-}} g_{2}^{(2 k)}(y)-\lim _{x \rightarrow 1^{-}} g_{3}^{(2 k)}(x)\right), \\
& \alpha_{k 4}=\frac{2}{\pi(2 k) !}\left((-1)^{k} \lim _{x \rightarrow 0^{+}} g_{3}^{(2 k)}(x)-\lim _{y \rightarrow 1^{-}} g_{4}^{(2 k)}(y)\right) .
\end{aligned}
$$

Then $u_{k}$ satisfies

$$
\begin{cases}\Delta u_{k}=0 & \text { in } \Omega, \\ \left.u_{k}\right|_{\partial \Omega}=g-\left.\sum_{i=1}^{4} \alpha_{k i} V_{k}^{i}\right|_{\partial \Omega} & \text { on } \partial \Omega,\end{cases}
$$

and the boundary data $\left.u_{k}\right|_{\partial \Omega}$ has property $A_{k}$.

By induction, for any $k \geq 0$, we can therefore convert the general problem (1.1) to one of the same form having property $A_{k}$.

\section{Conversion to a BVP with Property $B_{n}$}

In the last section, we gave a method of converting a general boundary value problem to one which has property $A_{n}$. Here we convert the problem further to one for which the even-order derivatives of the boundary data vanish at all four corners, up to a certain order, i.e., yielding property $B_{n}$ for some $n$. A BVP with property $B_{n}$ for some large $n$ does have fast-converging Fourier series solutions. We need two lemmas:

Lemma 3.1. For any $k \geq 0$, the polynomial

$$
W_{k}(x, y)=\sum_{i=0}^{k} \frac{(-1)^{i}}{(2(k-i)+1) !(2 i+1) !} x^{2(k-i)+1} y^{2 i+1}, \quad k \geq 0,
$$

has the following properties:

(1) $W_{k}(x, y)$ is harmonic. Also the restriction $\left.W_{k}\right|_{\partial \Omega}$ has property $b_{k}$ at $(0,0),(1,0),(0,1)$, but not at $(1,1)$.

(2) $\partial^{2 k} W_{k} / \partial x^{2 k}=x y$ and $\partial^{2 k} W_{k} / \partial y^{2 k}=(-1)^{k} x y$.

(3) $\partial^{2} W_{k}(x, y) / \partial x^{2}=-\partial^{2} W_{k}(x, y) / \partial y^{2}=W_{k-1}(x, y)$ for $k \geq 1$.

Proof. These properties can be verified directly.

Lemma 3.2. A problem (1.1) which has property $A_{k}$ for some $k \geq 0$ can be converted to a problem of the same form which has property $B_{k}$ by subtracting from the solution of (1.1) a linear combination of the functions $W_{j}^{1}(x, y)=$ 
$W_{j}(x, y), W_{j}^{2}(x, y)=W_{j}(y, 1-x), W_{j}^{3}(x, y)=W_{j}(1-x, 1-y)$, and $W_{j}^{4}(x, y)=W_{j}(1-y, x), j=0,1, \ldots, k$.

Proof. Set $v_{0}=u-\sum_{i=1}^{4} \beta_{k i} W_{k}^{i}$, where

$$
\begin{aligned}
& \beta_{k 1}=\lim _{y \rightarrow 1^{-}} g_{2}^{(2 k)}(y)=(-1)^{k} \lim _{x \rightarrow 1^{-}} g_{3}^{(2 k)}(x), \\
& \beta_{k 2}=\lim _{x \rightarrow 0^{+}} g_{3}^{(2 k)}(x)=(-1)^{k} \lim _{y \rightarrow 1^{-}} g_{4}^{(2 k)}(y), \\
& \beta_{k 3}=\lim _{y \rightarrow 0^{+}} g_{4}^{(2 k)}(y)=(-1)^{k} \lim _{x \rightarrow 0^{+}} g_{1}^{(2 k)}(x), \\
& \beta_{k 4}=\lim _{x \rightarrow 1^{-}} g_{1}^{(2 k)}(x)=(-1)^{k} \lim _{y \rightarrow 0^{+}} g_{2}^{(2 k)}(y) .
\end{aligned}
$$

Then $v_{0}$ satisfies

$$
\begin{cases}\Delta u_{0}=0 & \text { in } \Omega, \\ \left.u_{0}\right|_{\partial \Omega}=g-\left.\sum_{i=1}^{4} \beta_{k i} W_{k}^{i}\right|_{\partial \Omega} & \text { on } \partial \Omega,\end{cases}
$$

which has property $b_{k}$. Since $W_{k}^{i}(x, y)$ is a polynomial, all derivatives are well defined and continuous at the corners of $\Omega$, and since $W_{k}^{i}(x, y)$ is harmonic, the restriction of $W_{k}^{i}(x, y)$ to $\partial \Omega$ has property $A_{j}$ for arbitrary $j$. Therefore, problem (3.2) also has property $A_{k}$.

We apply this same procedure to convert (3.2) to a problem with property $b_{k-1}$ for $v_{1}=v_{0}-\sum_{i=1}^{4} \beta_{k-1, i} W_{k-1}^{i}$, which also has property $b_{k}$, since the derivatives of $W_{k-1}^{i}$ of order $2 k$ vanish identically. Continuing in this way, we find a problem for $v_{k}=v_{k-1}-\sum_{i=1}^{4} \beta_{0, i} W_{0}^{i}$ which has properties $b_{0}, b_{1}, \cdots, b_{k}$, and so has property $B_{k}$ as desired.

The following theorem describes a systematic way to convert a general system (1.1) to one with property $B_{n}$.

Theorem 3.1. For any fixed $n \geq 0$, a general Laplace's equation (1.1) can be converted to one in the same form which has property $B_{n}$; the conversion can be performed in the following steps:

(1) Set $U(x, y)=u-\sum_{k=0}^{n} \sum_{i=1}^{4}\left(c_{k i} V_{k}^{i}+d_{k i} W_{k}^{i}\right)$, where $c_{k i}$ and $d_{k i}$ are $8(n+1)$ undetermined coefficients.

(2) Set up 8(n+1) linear equations, i.e., for $j=0, \ldots, n$ let

$$
\begin{aligned}
& \lim _{x \rightarrow 0^{+}}\left(\left.U\right|_{\partial \Omega_{1}}\right)^{(2 j)}(x)=\lim _{y \rightarrow 0^{+}}\left(\left.U\right|_{\partial \Omega_{4}}\right)^{(2 j)}(y)=0, \\
& \lim _{x \rightarrow 1^{-}}\left(\left.U\right|_{\partial \Omega_{1}}\right)^{(2 j)}(x)=\lim _{y \rightarrow 0^{+}}\left(\left.U\right|_{\partial \Omega_{2}}\right)^{(2 j)}(y)=0, \\
& \lim _{x \rightarrow 1^{-}}\left(\left.U\right|_{\partial \Omega_{3}}\right)^{(2 j)}(x)=\lim _{y \rightarrow 1^{-}}\left(\left.U\right|_{\partial \Omega_{2}}\right)^{(2 j)}(y)=0, \\
& \lim _{x \rightarrow 0^{+}}\left(\left.U\right|_{\partial \Omega_{3}}\right)^{(2 j)}(x)=\lim _{y \rightarrow 1^{-}}\left(\left.U\right|_{\partial \Omega_{4}}\right)^{(2 j)}(y)=0 .
\end{aligned}
$$


(3) Solve the linear system for the $8(n+1)$ coefficients $c_{k i}, d_{k i}$ (there is a unique solution).

Then the new BVP:

$$
\left\{\begin{array}{l}
\Delta U=0, \\
\left.U\right|_{\partial \Omega_{j}}=g_{j}-\left[\sum_{k=0}^{n} \sum_{i=1}^{4}\left(c_{k i} V_{k j}^{i}+d_{k i} W_{k j}^{i}\right)\right], \quad j=1,2,3,4,
\end{array}\right.
$$

has property $B_{n}$. Here, $V_{k}^{i}=V_{k}^{i}(x, y)$ and $W_{k}^{i}=W_{k}^{i}(x, y), i=1,2,3,4$, are defined in Lemmas 2.1 and 3.2, and $V_{k j}^{i}$ denotes the restriction of $V_{k}^{i}(x, y)$ to the $j$ th boundary $(j=1,2,3,4)$.

Proof. Consider applying Lemmas 2.1 and 3.2 to a general problem (1.1). Boundary data $g$ can be constructed with arbitrary values for each of the $8(n+1)$ limits $\lim _{x \rightarrow 0^{+}} g_{1}^{(2 j)}(x), \quad \lim _{x \rightarrow 1^{-}} g_{1}^{(2 j)}(x), \lim _{y \rightarrow 0^{+}} g_{2}^{(2 j)}(y)$, $\lim _{y \rightarrow 1^{-}} g_{2}^{(2 j)}(y), \quad \lim _{x \rightarrow 0^{+}} g_{3}^{(2 j)}(x), \quad \lim _{x \rightarrow 1^{-}} g_{3}^{(2 j)}(x), \quad \lim _{y \rightarrow 0^{+}} g_{4}^{(2 j)}(y)$, $\lim _{y \rightarrow 1^{-}} g_{4}^{(2 j)}(y)$, for $j=0, \ldots, n$. The application of Lemmas 2.1 and 3.2 constructs a new BVP with property $B_{n}$ such that the difference between the solution of the original problem (1.1) and the new problem is a linear combination

$$
\sum_{k=0}^{n} \sum_{i=1}^{4}\left(c_{k i} V_{k}^{i}(x, y)+d_{k i} W_{k}^{i}(x, y)\right) .
$$

The combination coefficients $c_{k i}, d_{k i}, k=0, \ldots, n, i=1, \ldots, 4$, provide a solution of the $8(n+1)$ linear equations described in step 2 above. Since there exists such a solution vector in $R^{8(n+1)}$ for arbitrary forcing vector in $R^{8(n+1)}$, the coefficient matrix of the linear system is necessarily nonsingular and the solution for any particular boundary data is unique.

\section{COEFFICIENTS OF FOURIER SERIES SOLUTIONS AND TRUNCATION ERROR}

We rewrite (3.3) as the BVP,

$$
\left\{\begin{array}{l}
\Delta U=0, \\
\left.U\right|_{\partial \Omega_{j}}=G_{j}, \quad j=1,2,3,4,
\end{array}\right.
$$

which has property $B_{n}$.

In this section we solve (4.1) by solving four problems, each a Laplace equation with boundary function nonzero only on one side of the rectangle. Classical Fourier series methods are used to solve each of the four problems.

4.1. The four boundary value problems. Consider

$$
\left\{\begin{array}{l}
\Delta U=0, \\
\left.U\right|_{\partial \Omega_{j}}=G_{j}, \\
\left.U\right|_{\partial \Omega_{k}}=0,
\end{array} \quad k \neq j,\right.
$$


for $j=1,2,3,4$. The Fourier series solutions are

$$
\begin{aligned}
& U_{1}(x, y)=\sum_{m=1}^{\infty} a_{1 m} \frac{\sinh m \pi(1-y)}{\sinh m \pi} \sin m \pi x, \\
& U_{2}(x, y)=\sum_{m=1}^{\infty} a_{2 m} \frac{\sinh m \pi x}{\sinh m \pi} \sin m \pi y, \\
& U_{3}(x, y)=\sum_{m=1}^{\infty} a_{3 m} \frac{\sinh m \pi y}{\sinh m \pi} \sin m \pi x, \\
& U_{4}(x, y)=\sum_{m=1}^{\infty} a_{4 m} \frac{\sinh m \pi(1-x)}{\sinh m \pi} \sin m \pi y,
\end{aligned}
$$

where

$$
\begin{aligned}
a_{j m} & =2 \int_{0}^{1} G_{j} \sin m \pi s d s \\
& =2\left[\int_{0}^{1} g_{j} \sin m \pi s d s\right. \\
& \left.\quad-\sum_{k=0}^{n} \sum_{i=1}^{4}\left(c_{k i} \int_{0}^{1} V_{k j}^{i} \sin m \pi s d s+d_{k i} \int_{0}^{1} W_{k j}^{i} \sin m \pi s d s\right)\right]
\end{aligned}
$$

for $j=1,2,3,4$. The computation of $\int_{0}^{1} V_{k j}^{i} \sin m \pi s d s$ and $\int_{0}^{1} W_{k j}^{i} \sin m \pi s d s$ for $i, j=1,2,3,4$, is described in Appendix A and B.

4.2. Error from truncating the Fourier series solutions. Approximate solutions of $(4.2), j=1, \ldots, 4$, are given by

$$
\begin{aligned}
& U_{1}^{N}(x, y)=\sum_{m=1}^{N} a_{1 m} \frac{\sinh m \pi(1-y)}{\sinh m \pi} \sin m \pi x, \\
& U_{2}^{N}(x, y)=\sum_{m=1}^{N} a_{2 m} \frac{\sinh m \pi x}{\sinh m \pi} \sin m \pi y, \\
& U_{3}^{N}(x, y)=\sum_{m=1}^{N} a_{3 m} \frac{\sinh m \pi y}{\sinh m \pi} \sin m \pi x, \\
& U_{4}^{N}(x, y)=\sum_{m=1}^{N} a_{4 m} \frac{\sinh m \pi(1-x)}{\sinh m \pi} \sin m \pi y .
\end{aligned}
$$

Since (4.1) has property $B_{n}$, it follows from integration by parts that

$$
\begin{aligned}
a_{j m} & =2 \int_{0}^{1} G_{j} \sin m \pi s d s \\
& =\frac{2(-1)^{n+1}}{(m \pi)^{2 n+2}} \int_{0}^{1} G_{j}^{(2 n+2)}(s) \sin m \pi s d s
\end{aligned}
$$


for each $j=1,2,3,4$. Let $M_{j n}$ be such that $\max _{0 \leq s \leq 1}\left|G_{j}^{(2 n+2)}(s)\right| \leq M_{j n}$. Since the original boundary data is elementary, the bound $M_{j n}$ can be computed using interval arithmetic and Taylor series methods. Then

$$
\left|a_{j m}\right| \leq \frac{2 M_{j n}}{(m \pi)^{2 n+2}}
$$

and

$$
\begin{aligned}
\left|U_{j}(x, y)-U_{j}^{N}(x, y)\right| & \leq \frac{2 M_{j n}}{\pi^{2 n+2}} \int_{N}^{\infty} \frac{d x}{x^{2 n+2}} \\
& =\frac{2 M_{j n}}{(2 n+1) \pi^{2 n+2} N^{2 n+1}} .
\end{aligned}
$$

Therefore $U^{N}(x, y)=\sum_{j=1}^{4} U_{j}^{N}(x, y)$ is an approximate solution of (4.1) such that

$$
\left|U(x, y)-U^{N}(x, y)\right| \leq \epsilon_{n, N}^{\text {trunc }},
$$

where

$$
\epsilon_{n, N}^{\text {trunc }}=\frac{2\left(M_{1 n}+M_{2 n}+M_{3 n}+M_{4 n}\right)}{(2 n+1) \pi^{2 n+2} N^{2 n+1}} .
$$

\section{AN EXAMPLE OF THE ELIMINATION METHOD}

Consider

$$
\begin{cases}\Delta u=0 \text { in } \Omega=(0,1) \times(0,1) ; \\ u(x, 0)=g_{1}(x)=x^{2}, & 0 \leq x<1, \\ u(1, y)=g_{2}(y)=0, & 0 \leq y<1, \\ u(x, 1)=g_{3}(x)=0, & 0 \leq x<1, \\ u(0, y)=g_{4}(y)=0, & 0 \leq y<1 .\end{cases}
$$

From the boundary data $g$ it follows that (5.1) does not have property $A_{0}$. We set $u_{0}=u-\sum_{i=1}^{4} \alpha_{0 i} V_{0}^{i}$, where $\alpha_{01}=0, \alpha_{02}=\frac{2}{\pi}, \alpha_{03}=0$, and $\alpha_{04}=0$ are determined by $(2.3)$. Then $u_{0}$ satisfies

$$
\left\{\begin{array}{l}
\Delta u_{0}=0 \text { in } \Omega=(0,1) \times(0,1) ; \\
u_{0}(x, 0)=g_{01}(x)=x^{2}-1, \quad 0<x<1, \\
u_{0}(1, y)=g_{02}(y)=0, \quad 0<y<1, \quad 0<x<1, \\
u_{0}(x, 1)=g_{03}(x)=-\frac{2}{\pi} \arctan (1-x), \quad 0<0<1, \\
u_{0}(0, y)=g_{04}(y)=-\frac{2}{\pi} \arctan \left(\frac{1}{y}\right), \quad 0<y<
\end{array}\right.
$$

so (5.2) has property $A_{0}$.The new boundary data is continuous, and the second derivatives of the boundary data $\left.u_{0}\right|_{\partial \Omega}$ are given by

$$
\left\{\begin{array}{l}
g_{01}^{\prime \prime}(x)=2 \\
g_{02}^{\prime \prime}(y)=0 \\
g_{03}^{\prime \prime}(x)=\frac{4(1-x)}{\pi\left[1+(1-x)^{2}\right]^{2}} \\
g_{04}^{\prime \prime}(y)=-\frac{4 y}{\pi\left(1+y^{2}\right)^{2}}
\end{array}\right.
$$


It follows that problem (5.2) does not have property $A_{1}$ (condition (1.4) is violated). We set $u_{1}=u_{0}-\sum_{i=1}^{4} \alpha_{1 i} V_{1}^{i}$, where $\alpha_{11}=-\frac{2}{\pi}, \alpha_{12}=-\frac{2}{\pi}, \alpha_{13}=0$, and $\alpha_{14}=0$ are determined by (2.4) for $k=1$. Then $u_{1}$ satisfies

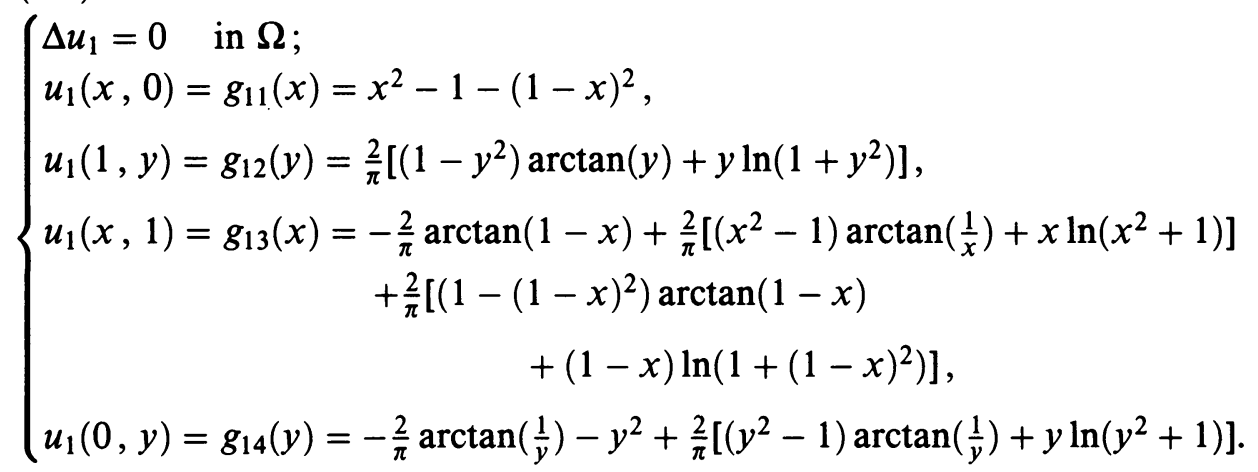

The new boundary data $\left.u_{1}\right|_{\partial \Omega}$ is continuous, and the second derivatives satisfy (1.4) for $j=1$, so (5.3) has property $A_{1}$. Continuing in this way, we set $u_{2}=u_{1}-\sum_{i=1}^{4} \alpha_{2 i} V_{2}^{i}$, where $\alpha_{21}=\alpha_{22}=\alpha_{23}=\alpha_{24}=0$ are determined by (2.4) for $k=2$, so $u_{2}$ satisfies the system

$$
\left\{\begin{array}{l}
\Delta u_{2}=0 \quad \text { in } \Omega \\
\left.u_{2}\right|_{\partial \Omega}=\left.\left(u-\frac{2}{\pi} V_{0}^{2}+\frac{2}{\pi} V_{1}^{1}+\frac{2}{\pi} V_{1}^{2}\right)\right|_{\partial \Omega}
\end{array}\right.
$$

(identical with (5.3)), which has property $A_{2}$. Now we convert (5.4) into a system with property $B_{2}$. We start by setting $v_{0}=u_{2}-\sum_{i=1}^{4} \beta_{2 i} W_{2}^{i}$, where $\beta_{21}=\beta_{22}=\frac{2}{\pi}, \beta_{23}=\beta_{24}=0$ are determined by (3.1) for $k=2$. Then $v_{0}$ satisfies

$$
\left\{\begin{array}{l}
\Delta v_{0}=0 \text { in } \Omega \\
\left.v_{0}\right|_{\partial \Omega}=\left.\left(u_{2}-\frac{2}{\pi} W_{2}^{1}(x, y)-\frac{2}{\pi} W_{2}^{2}(x, y)\right)\right|_{\partial \Omega}
\end{array}\right.
$$

which has property $b_{2}$. That is, the fourth-order derivatives of the boundary data vanish at the corners. Next, we set $v_{1}=v_{0}-\sum_{i=1}^{4} \beta_{1 i} W_{1}^{i}$, where $\beta_{11}=1$, $\beta_{12}=-\frac{1+\pi}{\pi}, \beta_{13}=\beta_{14}=0$ are from (3.1) for $k=1$. Then $v_{1}$ satisfies

$$
\left\{\begin{array}{l}
\Delta v_{1}=0 \quad \text { in } \Omega, \\
\left.v_{1}\right|_{\partial \Omega}=\left.\left(u_{2}-\frac{2}{\pi} W_{2}^{1}(x, y)-\frac{2}{\pi} W_{2}^{2}(x, y)-W_{1}^{1}(x, y)+\left(1+\frac{1}{\pi}\right) W_{1}^{2}(x, y)\right)\right|_{\partial \Omega},
\end{array}\right.
$$

which has both properties $b_{2}$ and $b_{1}$. Finally, we set $v_{2}=v_{1}-\sum_{i=1}^{4} \beta_{0 i} W_{0}^{i}$, where $\beta_{01}=\frac{90 \ln 2+1}{45 \pi}, \beta_{02}=-\frac{135 \pi-180 \ln 2-2}{90 \pi}, \beta_{03}=-2$, and $\beta_{04}=0$ are from 
(3.1) for $k=0$. Then $v_{2}$ satisfies

$$
\left\{\begin{array}{l}
\Delta v_{2}=0 \quad \text { in } \Omega \\
\left.v_{2}\right|_{\partial \Omega}=\left(u_{2}-\frac{2}{\pi} W_{2}^{1}(x, y)-\frac{2}{\pi} W_{2}^{2}(x, y)-W_{1}^{1}(x, y)\right. \\
+\left(1+\frac{1}{\pi}\right) W_{1}^{2}(x, y)-\frac{90 \ln 2+1}{45 \pi} W_{0}^{1} \\
\left.\quad+\frac{135 \pi-180 \ln 2-2}{90 \pi} W_{0}^{2}+2 W_{0}^{3}\right)\left.\right|_{\partial \Omega}
\end{array}\right.
$$

Now problem (5.7) has property $B_{2}$, that is, properties $b_{2}, b_{1}$, and $b_{0}$ combined. As in $\S \S 3$ and 4 , let $U=v_{2}$. Then the solution $u$ of $(5.1)$ is given in terms of $U$ by

$$
u=U+\sum_{k=0}^{2} \sum_{i=1}^{4}\left(c_{k i} V_{k}^{i}+d_{k i} W_{k}^{i}\right)
$$

where

$$
\begin{aligned}
& c_{01}=0, c_{02}=\frac{2}{\pi}, c_{03}=c_{04}=0, \\
& c_{11}=c_{12}=-\frac{2}{\pi}, c_{13}=c_{24}=0, \\
& c_{21}=c_{22}=c_{23}=c_{24}=0 ; \\
& d_{01}=\frac{90 \ln 2+1}{45 \pi}, d_{02}=-\frac{135 \pi-180 \ln 2-2}{90 \pi}, d_{03}=-2, d_{04}=0, \\
& d_{11}=1, d_{12}=-\left(1+\frac{1}{\pi}\right), d_{13}=d_{14}=0, \\
& d_{21}=d_{22}=\frac{2}{\pi}, d_{23}=d_{24}=0 .
\end{aligned}
$$

We let $U=U_{1}+U_{2}+U_{3}+U_{4}$, where each $U_{j}$ is harmonic and $\left.U_{j}\right|_{\partial \Omega_{k}}=0$, $k \neq j$. We solve for each $U_{j}$ by truncated Fourier series as in $\S 4$, taking $N=3$. Then $U$ is approximated by

$$
\begin{aligned}
U^{3}(x, y)= & \sum_{m=1}^{3} a_{1 m} \frac{\sinh m \pi(1-y)}{\sinh m \pi} \sin m \pi x+\sum_{m=1}^{3} a_{2 m} \frac{\sinh m \pi x}{\sinh m \pi} \sin m \pi y \\
& +\sum_{m=1}^{3} a_{3 m} \frac{\sinh m \pi y}{\sinh m \pi} \sin m \pi x+\sum_{m=1}^{3} a_{4 m} \frac{\sinh m \pi(1-x)}{\sinh m \pi} \sin m \pi y,
\end{aligned}
$$

where the coefficients $a_{j m}$ are given by

$$
\begin{aligned}
a_{j m}=2\left[\int_{0}^{1} g_{j}\right. & \sin m \pi s d s \\
& \left.\quad-\sum_{k=0}^{2} \sum_{i=1}^{4}\left(c_{k i} \int_{0}^{1} V_{k j}^{i} \sin m \pi s d s+d_{k i} \int_{0}^{1} W_{k j}^{i} \sin m \pi s d s\right)\right] .
\end{aligned}
$$

We computed the coefficients $a_{j m}, 1 \leq j \leq 4,1 \leq m \leq 3$, "precisely", using Aberth's interval arithmetic package. The integrals $\int_{0}^{1} V_{k j}^{i} \sin m \pi s d s$ and $\int_{0}^{1} W_{k j}^{i} \sin m \pi s d s$ were evaluated in terms of tabulated, precisely calculated values of the quantities $G_{i m}(1), G_{i m}(0), S(m, j)$, and $C(m, j)$ as in Appendix 
A. We found

$$
\begin{aligned}
& a_{11}=0.0000000 \\
& a_{12}=0.0000000 \\
& a_{13}=0.0000000 \\
& a_{21}=0.0051243 \\
& a_{22}=0.0000321 \\
& a_{23}=0.0000003 \\
& a_{31}=-0.0150388 \\
& a_{32}=0.0006332 \\
& a_{33}=-0.0000139 \\
& a_{41}=0.0304116 \\
& a_{42}=0.0006653 \\
& a_{43}=0.0000148
\end{aligned}
$$

Here, each of the values of the coefficients given is "precise" in the sense that every digit listed is correct. The roundoff error in each coefficient is less than $0.5 \times 10^{-7}$; the statement " $a_{21}=0.0051243$ " means that the actual value of $a_{21}$ lies in the interval [0.00512425, 0.00512435].

The error $\epsilon_{2,3}^{\text {trunc }}$ in the approximation

$$
U^{3}(x, y)+\sum_{k=0}^{2} \sum_{i=1}^{4}\left(c_{k i} V_{k}^{i}(x, y)+d_{k i} W_{k}^{i}(x, y)\right)
$$

due to truncating the Fourier series can be obtained from bounds on the 6thorder derivatives of the boundary data $G_{j}=\left.U\right|_{\partial \Omega}$ as in $\S 4$. Since the functions $W_{k}^{i}(x, y)$, when restricted to the boundaries, are polynomials of degree $2 k+1$, and $k \leq 2$, these functions are not involved in the bounds on $\left|G_{j}^{(6)}\right|$. The restrictions of $V_{k}^{i}(x, y)$ to the boundaries, as given by the formulas in Appendix $A$, are elementary functions of the boundary coordinate, except in some cases at the endpoints. In the exceptional cases, tangent identities can be used to give an alternative formula which is elementary at the particular endpoint.

We divided each boundary into a grid of 100 points and evaluated the boundary functions $G_{j}$ "as Taylor series" of degree 6 , with the independent variable representing an interval of length 0.01 . The coefficients of the Taylor series were then each interval-valued. A bound for $G_{j}^{(6)}$ over the particular subinterval was obtained from the Taylor coefficient of the 6 th power. We found that

$$
\begin{aligned}
& \max _{0 \leq x \leq 1}\left|G_{1}^{(6)}(x)\right| \leq M_{12}=0.0000000, \\
& \max _{0 \leq x \leq 1}\left|G_{2}^{(6)}(x)\right| \leq M_{22}=58.0000000, \\
& \max _{0 \leq x \leq 1}\left|G_{3}^{(6)}(x)\right| \leq M_{32}=130.0000000, \\
& \max _{0 \leq x \leq 1}\left|G_{4}^{(6)}(x)\right| \leq M_{42}=111.0000000 .
\end{aligned}
$$

Therefore, the truncation error is bounded by

$$
\epsilon_{n, N}^{\text {trunc }}=\epsilon_{2,3}^{\text {trunc }}=0.0005119479
$$


TABLE 1. The value of the approximate solution on a $9 \times 9$ grid

$y$

\begin{tabular}{|l|l|l|l|l|l|l|l|l|l|}
\hline & 0.1 & 0.2 & 0.3 & 0.4 & 0.5 & 0.6 & 0.7 & 0.8 & 0.9 \\
\hline 0.1 & 0.02241 & 0.02525 & 0.02377 & 0.02042 & 0.01648 & 0.01257 & 0.00898 & 0.00574 & 0.00279 \\
\hline 0.2 & 0.05331 & 0.05444 & 0.04923 & 0.04134 & 0.03285 & 0.02480 & 0.01758 & 0.01119 & 0.00543 \\
\hline 0.3 & 0.09598 & 0.08961 & 0.07711 & 0.06267 & 0.04867 & 0.03615 & 0.02533 & 0.01599 & 0.00772 \\
\hline 0.4 & 0.15074 & 0.13055 & 0.10661 & 0.08336 & 0.06289 & 0.04572 & 0.03155 & 0.01972 & 0.00947 \\
\hline 0.5 & 0.21606 & 0.17477 & 0.13501 & 0.10102 & 0.07367 & 0.05223 & 0.03541 & 0.02188 & 0.01043 \\
\hline 0.6 & 0.28801 & 0.21659 & 0.15710 & 0.11177 & 0.07848 & 0.05413 & 0.03600 & 0.02196 & 0.01040 \\
\hline 0.7 & 0.35750 & 0.24484 & 0.16436 & 0.11041 & 0.07443 & 0.04991 & 0.03257 & 0.01964 & 0.00924 \\
\hline 0.8 & 0.40086 & 0.23810 & 0.14494 & 0.09151 & 0.05930 & 0.03877 & 0.02489 & 0.01485 & 0.00695 \\
\hline 0.9 & 0.34335 & 0.16149 & 0.08831 & 0.05281 & 0.03320 & 0.02131 & 0.01352 & 0.00801 & 0.00373 \\
\hline
\end{tabular}

Table 1 gives the result of evaluating

$$
U^{3}(x, y)+\sum_{k=0}^{2} \sum_{i=1}^{4}\left(c_{k i} V_{k}^{i}(x, y)+d_{k i} W_{k}^{i}(x, y)\right)
$$

on a $9 \times 9$ grid. Interval arithmetic was used in this evaluation. The intervals include truncation error from the quadrature in evaluating the various coefficients as well as all roundoff error. All digits shown in Table 1 are correct; each value has an error at most $0.5 \times 10^{-5}$. For example, from the entry for $x=y=1 / 2$, we have

$$
\begin{aligned}
\left|u\left(\frac{1}{2}, \frac{1}{2}\right)-0.07367\right| & \leq 0.5 \times 10^{-5}+\epsilon_{2,3}^{\text {trunc }} \\
& \leq 0.6 \times 10^{-3},
\end{aligned}
$$

and so $u\left(\frac{1}{2}, \frac{1}{2}\right) \in[0.07307,0.07427]$. Similar computations lead to true bounds for the exact solution $u(x, y)$ at the other grid points.

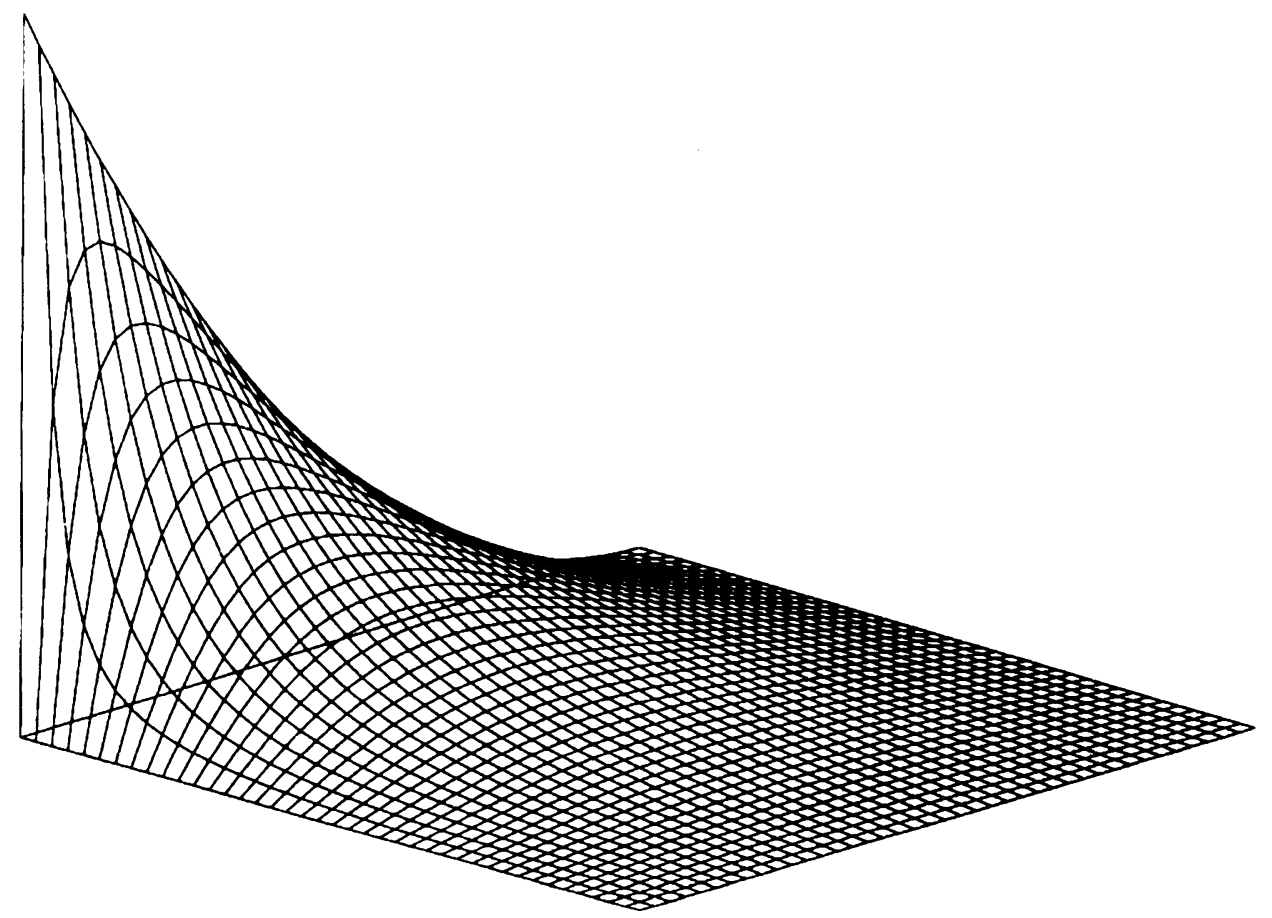

Figure 1. Solution of (5.1) 
Figure 1 is a graph of the solution, based on values of the approximate solution on a $40 \times 40$ grid. One unit of the solution corresponds to $2^{\prime \prime}$ on the graph, so $\epsilon_{2,3}$ corresponds to roughly $.001^{\prime \prime}$ on the plot, which was done on a 300dpi laser printer. The total error at grid points in this figure is therefore at most one pixel. Between grid points, the graph is constructed by linear interpolation. The graph shown therefore is uniformly within one pixel width of the graph of the true solution, constructed by linear interpolation on the same grid. The main source of error present in Figure 1 is the use of linear interpolation between grid points. This could be reduced to less than one pixel by evaluating the approximate solution on a finer mesh.

\section{Discussion}

Work related to ours is due to J. Barkley Rosser [9, 10], who developed schemes for solving Laplace's equation in regions with re-entrant corners, for example L-shaped and T-shaped regions. Such corners "cause" difficulties with the convergence rate of traditional finite difference and finite element methods. Rosser developed hybrid schemes which cure such difficulties. Near each re-entrant corner, these schemes employ approximations based on harmonic functions in sectors, constructed to match boundary data specified on radii. Although the problem we consider has no re-entrant corners, our scheme resembles Rosser's in that we regard each of the four corners as a source of convergence difficulties, much as Rosser regards the re-entrant corners. To obtain a function harmonic in a sector and which satisfies prescribed boundary data on radii, Rosser employed conformal mapping. This harmonic function is somewhat analogous to our linear combination $\sum_{k=0}^{n} \sum_{i=1}^{4}\left(c_{k i} V_{k}^{i}+d_{k i} W_{k}^{i}\right)$. Instead of matching the boundary data, however, our linear combination matches just the even-order radial derivatives of the boundary data at the corners.

We note that for $\alpha=\frac{\pi}{4}$, the functions $r^{\frac{m}{2 \alpha}} \sin \frac{\theta m}{2 \alpha}, m=1,2, \ldots$, which appear in a series in Rosser's work, reduce to our polynomials $W_{k}(x, y), k=$ $1,2, \ldots$.

In Rosser's work, the convergence rate of the hybrid scheme is that characteristic of the finite difference or finite element method used in conjunction with the re-entrant corner approximation scheme. Since we wished to perform "precise" computations, in our work we restricted attention to schemes which could provide extremely fast convergence rates and therefore did not consider finite difference or finite element methods.

The approximate scheme for (1.1) with general elementary boundary data has been implemented using the Aberth-Schaefer's " $\mathrm{C}++$ Module for Range Arithmetic" routine library. The program was written for AT\&T's C++ version 2.1 on a Sun Sparc station running SunOS4.1. The program also runs under MS-DOS 5.0 using Gnu's C++ version 1.05.

Input to the program are analytical expressions for the boundary functions, the integer $n$ which determines the order $2 n$ to which the derivatives of the boundary data for $u$ vanish at the corners, and the integer $N$ which determines the number of terms in the Fourier series part in the solution. Output are coefficients for the approximate solutions in terms of range numbers which are guaranteed to be correct to the last printed digit and also the bound $\epsilon_{n, N}^{\text {trunc }}$ on the error from truncating the Fourier series. 
The method we described above for solving Laplace's equation precisely can be used in solving the general Poisson's equation on the square precisely. Consider Poisson's equation:

$$
\left\{\begin{aligned}
\Delta u & =-F & & \text { in } \Omega \\
\left.u\right|_{\partial \Omega} & =f & & \text { on } \partial \Omega
\end{aligned}\right.
$$

where $\Omega$ is the unit square $(0,1) \times(0,1)$ and $\partial \Omega$ is the boundary of $\Omega$. We suppose that $F(x, y)$ is a continuous elementary function and $f$ is defined on the sides $\partial \Omega_{j}, j=1,2,3,4$, of the square by continuous elementary functions $f_{1}(x), f_{2}(y), f_{3}(x)$, and $f_{4}(y)$. The idea is to convert (6.1) to a Laplace equation precisely; then we can use the method we have developed. The conversion can be done as follows.

We can first prove a Chebyshev-Lagrange interpolation theorem which gives a polynomial approximation to an arbitrary elementary function $F$ of two variables. As the error bounds for the interpolation are given in terms of bounds on the partial derivatives of $F$, and $F$ is elementary, we can compute error bounds using Taylor series methods and interval analysis.

We then apply Chebyshev-Lagrange interpolation to the forcing term $F$ in Poisson's equation, approximate the boundary value problem for Poisson's equation with forcing term $F$ by a similar problem but with polynomial forcing term. Computable bounds for the error introduced in the conversion are derived, using the maximum (minimum) principle.

Thirdly, we derive formulas for a particular solution of Poisson's equation with the polynomial forcing term. Using this particular solution, the boundary value problem for Poisson's equation is converted to one for a Laplace equation. The conversion is exact, and the boundary conditions of the converted problem are still elementary functions.

The particular solution to Poisson's equation

$$
\Delta \omega=x^{i} y^{j}
$$

is

$$
\omega_{i j}(x, y)=\sum_{k=1}^{\left[\frac{k}{2}\right]+1}(-1)^{k+1} \frac{A_{j}^{2 k-2}}{A_{i+2 k}^{2 k}} x^{i+2 k} y^{j-2 k+2},
$$

where $A_{n}^{k}=n(n-1) \cdots(n-k+1)$.

We expect that the same basic ideas can also be extended to solve Helmholz's equation precisely. This is more difficult, but we have found functions with which to extract corner singularities for Helmholz's equation.

\section{APPENDIX A. FOURIER COEFFICIENTS OF $V_{k 1}^{i}$}

A.1. Computing $\int_{0}^{1} V_{k 1}^{i} \sin m \pi x d x$. Recall $V_{k j}^{i}=\left.V_{k}^{i}(x, y)\right|_{\partial \Omega_{j}}$ from $\S 4$, where $V_{k}^{i}$ is defined in Lemma 2.1. We have four functions: 


$$
\begin{aligned}
V_{k 1}^{1} \equiv & 0, \\
V_{k 1}^{2}= & \frac{\pi}{2}(-1)^{k}(1-x)^{2 k}, \\
V_{k 1}^{3}= & \left(1+(1-x)^{2}\right)^{k} \cos \left(2 k \arctan \frac{1}{1-x}\right) \arctan \frac{1}{1-x} \\
& +\frac{1}{2}\left(1+(1-x)^{2}\right)^{k} \sin \left(2 k \arctan \frac{1}{1-x}\right) \ln \left(1+(1-x)^{2}\right), \\
V_{k 1}^{4}= & \left(1+x^{2}\right)^{k} \cos (2 k \arctan x) \arctan x+\frac{1}{2}\left(1+x^{2}\right)^{k} \sin (2 k \arctan x) \ln \left(1+x^{2}\right) .
\end{aligned}
$$

For the second function,

$$
\begin{aligned}
\int_{0}^{1} V_{k 1}^{2}(x) \sin m \pi x d x & =\frac{\pi}{2}(-1)^{k} \int_{0}^{1}(1-x)^{2 k} \sin m \pi x d x \\
& =\frac{\pi}{2}(-1)^{k} \int_{0}^{1} x^{2 k} \sin (m \pi-m \pi x) d x \\
& =-\frac{\pi}{2}(-1)^{k} \int_{0}^{1} x^{2 k} \cos m \pi \sin m \pi x d x \\
& =\frac{\pi}{2}(-1)^{k+m+1} \int_{0}^{1} x^{2 k} \sin m \pi x d x \\
& =\frac{(2 k) !}{2 \pi^{2 k} m^{2 k+1}}\left[\sum_{i=0}^{k} \frac{(-1)^{k+i}}{(2 k-2 i) !}(m \pi)^{2 k-2 i}+(-1)^{m+1}\right] .
\end{aligned}
$$

To compute $\int_{0}^{1} V_{k 1}^{i}(x) \sin m \pi x d x, i=3$, 4, we need the following.

Lemma A.1. Let

$$
C_{k}(x)=\left(1+x^{2}\right)^{k} \cos (2 k \arctan x)
$$

and

$$
S_{k}(x)=\left(1+x^{2}\right)^{k} \sin (2 k \arctan x) .
$$

Then $C_{k}(x)$ and $S_{k}(x)$ are polynomials of degree $2 k$ and $2 k-1$, respectively.

Proof. With $T_{2 k}(x)$ denoting the Chebyshev polynomial of degree $2 k$, we have $T_{2 k}(\cos \theta)=\cos 2 k \theta$, hence

$$
\begin{aligned}
C_{k}(x) & =\left(1+x^{2}\right)^{k} \cos (2 k \arctan x)=\left(1+x^{2}\right)^{k} T_{2 k}(\cos (\arctan x)) \\
& =\left(1+x^{2}\right)^{k} T_{2 k}\left(\cos \left(\arccos \frac{1}{\sqrt{1+x^{2}}}\right)\right)=\left(1+x^{2}\right)^{k} T_{2 k}\left(\frac{1}{\sqrt{1+x^{2}}}\right),
\end{aligned}
$$

from which the first assertion follows.

Similarly, with $U_{2 k-1}(x)$ denoting the Chebyshev polynomial of the second kind, we have $U_{2 k-1}(\cos \theta)=\sin 2 k \theta / \sin \theta$, hence 


$$
\begin{aligned}
S_{k}(x) & =\left(1+x^{2}\right)^{k} \sin (2 k \arctan x)=\left(1+x^{2}\right)^{k} \sin (\arctan x) U_{2 k-1}(\cos (\arctan x)) \\
& =\left(1+x^{2}\right)^{k} \sin \left(\arcsin \frac{x}{\sqrt{1+x^{2}}}\right) U_{2 k-1}\left(\cos \left(\arccos \frac{1}{\sqrt{1+x^{2}}}\right)\right) \\
& =x\left(1+x^{2}\right)^{k-1 / 2} U_{2 k-1}\left(\frac{1}{\sqrt{1+x^{2}}}\right),
\end{aligned}
$$

which yields the second assertion.

Now we have

$$
\begin{aligned}
\int_{0}^{1} V_{k 1}^{4}(x) \sin m \pi x d x= & \int_{0}^{1} C_{k}(x) \arctan x \sin m \pi x d x \\
& +\frac{1}{2} \int_{0}^{1} S_{k}(x) \ln \left(1+x^{2}\right) \sin m \pi x d x
\end{aligned}
$$

For computing the two integrals on the right-hand side, set

$$
\int x^{i} \sin m \pi x d x=G_{i m}(x) \text { for } i=0, \ldots, k,
$$

where ([6, formula 2.633 .1$])$

$$
G_{i m}(x)=-\sum_{l=0}^{i} l ! C_{i}^{l} \frac{x^{i-l}}{(m \pi)^{l+1}} \cos \left(m \pi x+\frac{1}{2} l \pi\right)
$$

Taking one term from $C_{k}(x)$ or $S_{k}(x)$ (by Lemma A.1, these functions are polynomials), we have

$$
\begin{aligned}
\int_{0}^{1} x^{i} \arctan x \sin m \pi x d x & =\left.G_{i m}(x) \arctan x\right|_{0} ^{1}-\int_{0}^{1} \frac{1}{1+x^{2}} G_{i m}(x) d x \\
& =G_{i m}(1) \frac{\pi}{4}-\int_{0}^{1} \frac{1}{1+x^{2}} G_{i m}(x) d x
\end{aligned}
$$

and

$$
\begin{aligned}
\frac{1}{2} \int_{0}^{1} x^{i} \ln \left(1+x^{2}\right) \sin m \pi x d x & =\left.\frac{1}{2} G_{i m}(x) \ln \left(1+x^{2}\right)\right|_{0} ^{1}-\int_{0}^{1} \frac{x}{1+x^{2}} G_{i m}(x) d x \\
& =G_{i m}(1) \frac{\ln 2}{2}-\int_{0}^{1} \frac{x}{1+x^{2}} G_{i m}(x) d x .
\end{aligned}
$$

Since $G_{i m}(x)$ is a linear combination of $x^{j} \sin m \pi x$ and $x^{j} \cos m \pi x \quad(j \leq i)$, we can evaluate the desired integrals in terms of the integrals

$$
\int_{0}^{1} \frac{x^{j}}{1+x^{2}} \sin m \pi x d x=: S(j, m)
$$

and

$$
\int_{0}^{1} \frac{x^{j}}{1+x^{2}} \cos m \pi x d x=: C(j, m)
$$

for $j=0, \ldots, i+1, i=0, \ldots, k$. 
Now consider the computation of $\int_{0}^{1} V_{k 1}^{3}(x) \sin m \pi x d x$.

Let $\tilde{C}_{k}(x)=\left(1+x^{2}\right)^{k} \cos \left(2 k \arctan \frac{1}{x}\right)$. Then as before,

$$
\tilde{C}_{k}(x)=\left(1+x^{2}\right)^{k} T_{2 k}\left(\cos \left(\arctan \frac{1}{x}\right)\right)=\left(1+x^{2}\right)^{k} T_{2 k}\left(\frac{x}{\sqrt{1+x^{2}}}\right),
$$

i.e., $\tilde{C}_{k}(x)$ is a polynomial of degree $2 k$. Similarly, we can prove that the function $\tilde{S}_{k}(x)=\left(1+x^{2}\right)^{k} \sin \left(2 k\right.$ arctan $\left.\frac{1}{x}\right)$ is a polynomial of degree $2 k-1$. Therefore,

$$
\begin{aligned}
\int_{0}^{1} V_{k 1}^{3}(x) \sin m \pi x d x= & \int_{0}^{1} \tilde{C}_{k}(1-x) \arctan \frac{1}{1-x} \sin m \pi x d x \\
& +\frac{1}{2} \int_{0}^{1} \tilde{S}_{k}(1-x) \ln \left(1+(1-x)^{2}\right) \sin m \pi x d x \\
= & \int_{0}^{1} \tilde{C}_{k}(x) \arctan \frac{1}{x} \sin (m \pi-m \pi x) d x \\
& +\frac{1}{2} \int_{0}^{1} \tilde{S}_{k}(x) \ln \left(1+x^{2}\right) \sin (m \pi-m \pi x) d x \\
= & (-1)^{m+1} \int_{0}^{1} \tilde{C}_{k}(x) \arctan \frac{1}{x} \sin m \pi x d x \\
& +\frac{(-1)^{m+1}}{2} \int_{0}^{1} \tilde{S}_{k}(x) \ln \left(1+x^{2}\right) \sin m \pi x d x
\end{aligned}
$$

Taking one term from $\tilde{S}_{k}(x)$ or $\tilde{C}_{k}(x)$, we have

$$
\begin{aligned}
\int_{0}^{1} x^{i} \arctan \frac{1}{x} \sin m \pi x d x & =\left.G_{i m}(x) \arctan \frac{1}{x}\right|_{0} ^{1}+\int_{0}^{1} \frac{1}{1+x^{2}} G_{i m}(x) d x \\
& =G_{i m}(1) \frac{\pi}{4}-G_{i m}(0) \frac{\pi}{2}+\int_{0}^{1} \frac{1}{1+x^{2}} G_{i m}(x) d x
\end{aligned}
$$

and

$$
\frac{1}{2} \int_{0}^{1} x^{i} \ln \left(1+x^{2}\right) \sin m \pi x d x=G_{i m}(1) \frac{\ln 2}{2}-\int_{0}^{1} \frac{x}{1+x^{2}} G_{i m}(x) d x
$$

as before. So the integral can be also evaluated in terms of $S(j, m)$ and $C(j, m)$.

A.2. Computing $\int_{0}^{1} V_{k 2}^{i} \sin m \pi x d x$. From $\S 2$ we have

$$
\begin{aligned}
V_{k 2}^{1}(y) & =\left.V_{k}(x, y)\right|_{x=1}=V_{k}(1, y) \\
& =\left(1+y^{2}\right)^{k}\left(\cos (2 k \arctan y) \arctan y+\frac{1}{2} \sin (2 k \arctan y) \ln \left(1+y^{2}\right)\right), \\
V_{k 2}^{2}(y) & =\left.V_{k}^{2}(x, y)\right|_{x=1}=\left.V_{k}(y, 1-x)\right|_{x=1}=V_{k}(y, 0) \\
& \equiv 0
\end{aligned}
$$




$$
\begin{aligned}
V_{k 2}^{3}(y)= & \left.V_{k}^{3}(x, y)\right|_{x=1}=\left.V_{k}(1-x, 1-y)\right|_{x=1}=V_{k}(0,1-y) \\
= & (1-y)^{2 k}(-1)^{k} \frac{\pi}{2} \\
V_{k 2}^{4}(y)= & \left.V_{k}^{4}(x, y)\right|_{x=1}=\left.V_{k}(1-y, x)\right|_{x=1}=V_{k}(1-y, 1) \\
= & \left(1+(1-y)^{2}\right)^{k} \cos \left(2 k \arctan \frac{1}{1-y}\right) \arctan \frac{1}{1-y} \\
& +\frac{1}{2}\left(1+(1-y)^{2}\right)^{k} \sin \left(2 k \arctan \frac{1}{1-y}\right) \ln \left(1+(1-y)^{2}\right) .
\end{aligned}
$$

Then

$$
\int_{0}^{1} V_{k 2}^{1}(y) \sin m \pi y d y=\int_{0}^{1} V_{k 1}^{4}(x) \sin m \pi x d x
$$

which is evaluated when we compute $\int_{0}^{1} V_{k 1}^{4}(x) \sin m \pi x d x$, and similarly

$$
\begin{aligned}
& \int_{0}^{1} V_{k 2}^{3}(y) \sin m \pi y d y=\int_{0}^{1} V_{k 1}^{2}(x) \sin m \pi x d x \\
& \int_{0}^{1} V_{k 2}^{4}(y) \sin m \pi y d y=\int_{0}^{1} V_{k 1}^{3}(x) \sin m \pi x d x
\end{aligned}
$$

which are discussed in Appendix A.1.

A.3. Computing $\int_{0}^{1} V_{k 3}^{i} \sin m \pi x d x$. We have

$$
\begin{aligned}
V_{k 3}^{1}(x)= & \left.V_{k}(x, y)\right|_{y=1}=V_{k}(x, 1) \\
= & \left(1+x^{2}\right)^{k}\left(\cos \left(2 k \arctan \frac{1}{x}\right) \arctan \frac{1}{x}\right. \\
& \left.\quad+\frac{1}{2} \sin \left(2 k \arctan \frac{1}{x}\right) \ln \left(1+x^{2}\right)\right), \\
V_{k 3}^{2}(x)= & \left.V_{k}^{2}(x, y)\right|_{y=1}=\left.V_{k}(y, 1-x)\right|_{y=1}=V_{k}(1,1-x) \\
= & \left(1+(1-x)^{2}\right)^{k} \cos (2 k \arctan (1-x)) \arctan (1-x) \\
& +\frac{1}{2}\left(1+(1-x)^{2}\right)^{k} \sin (2 k \arctan (1-x)) \ln \left(1+(1-x)^{2}\right), \\
V_{k 3}^{3}(y)= & \left.V_{k}^{3}(x, y)\right|_{y=1}=\left.V_{k}(1-x, 1-y)\right|_{y=1}=V_{k}(1-x, 0) \\
& \equiv 0, \\
V_{k 3}^{4}(y)= & \left.V_{k}^{4}(x, y)\right|_{y=1}=\left.V_{k}(1-y, x)\right|_{y=1}=V_{k}(0, x) \\
= & x^{2 k}(-1)^{k} \frac{\pi}{2} .
\end{aligned}
$$

Then 


$$
\begin{aligned}
& \int_{0}^{1} V_{k 3}^{1}(x) \sin m \pi x d x \\
&= \int_{0}^{1}\left(1+x^{2}\right)^{k} \cos \left(2 k \arctan \frac{1}{x}\right) \arctan \frac{1}{x} \sin m \pi x d x \\
&+\frac{1}{2} \int_{0}^{1}\left(1+x^{2}\right)^{k} \sin \left(2 k \arctan \frac{1}{x}\right) \ln \left(1+x^{2}\right) \sin m \pi x d x \\
&= \int_{0}^{1} \tilde{C}_{k}(x) \arctan \frac{1}{x} \sin m \pi x d x \\
&+\frac{1}{2} \int_{0}^{1} \tilde{S}_{k}(x) \ln \left(1+x^{2}\right) \sin m \pi x d x \\
&=(-1)^{m+1} \int_{0}^{1} V_{k 1}^{3} \sin m \pi x d x, \\
& \int_{0}^{1} V_{k 3}^{2}(y) \sin m \pi y d y \\
&=\int_{0}^{1}\left(1+(1-x)^{2}\right)^{k} \cos (2 k \arctan (1-x)) \arctan (1-x) \sin m \pi x d x \\
&+\frac{1}{2}\left(1+(1-x)^{2}\right)^{k} \sin (2 k \arctan (1-x)) \ln \left(1+(1-x)^{2}\right) \sin m \pi x d x \\
&=(-1)^{m+1} \int_{0}^{1} V_{k 1}^{4}(x) \sin m \pi x d x, \\
& \int_{0}^{1} V_{k 3}^{4}(y) \sin m \pi y d y=\int_{0}^{1}(-1)^{k} \frac{\pi}{2} x^{2 k} \sin m \pi x d x \\
&=
\end{aligned}
$$

A.4. Computing $\int_{0}^{1} V_{k 4}^{i} \sin m \pi x d x$. We have

$$
\begin{aligned}
V_{k 4}^{1}(y)= & \left.V_{k}(x, y)\right|_{x=0}=V_{k}(0, y) \\
= & y^{2 k}(-1)^{k} \frac{\pi}{2}, \\
V_{k 4}^{2}(y)= & \left.V_{k}^{2}(x, y)\right|_{x=0}=\left.V_{k}(y, 1-x)\right|_{x=0}=V_{k}(y, 1) \\
= & \left(1+y^{2}\right)^{k} \cos \left(2 k \arctan \frac{1}{y}\right) \arctan \frac{1}{y} \\
V_{k 4}^{3}(y)= & \left.V_{k}^{3}(x, y)\right|_{x=0}=\left.V_{k}(1-x, 1-y)\right|_{x=0}=V_{k}(1,1-y) \\
= & \left(1+(1-y)^{2}\right)^{k} \cos (2 k \arctan (1-y)) \arctan (1-y) \\
& +\frac{1}{2}\left(1+(1-y)^{2}\right)^{k} \sin (2 k \arctan (1-y)) \ln \left(1+(1-y)^{2}\right), \\
V_{k 4}^{4}(y)= & \left.V_{k}^{4}(x, y)\right|_{x=0}=\left.V_{k}(1-y, x)\right|_{x=0}=V_{k}(1-y, 0) \\
\equiv & 0 .
\end{aligned}
$$

Then 


$$
\begin{aligned}
& \int_{0}^{1} V_{k 4}^{1}(y) \sin m \pi y d y=(-1)^{k} \frac{\pi}{2}\left(G_{2 k, m}(1)-G_{2 k, m}(0)\right), \\
& \int_{0}^{1} V_{k 4}^{2}(y) \sin m \pi y d y=\int_{0}^{1} V_{k 3}^{1}(x) \sin m \pi x d x, \\
& \int_{0}^{1} V_{k 4}^{3}(y) \sin m \pi y d y=\int_{0}^{1} V_{k 3}^{2}(x) \sin m \pi x d x,
\end{aligned}
$$

which are discussed in A.3.

The precise computation of the quantities $S(j, m)$ and $C(j, m)$ can be done by using Aberth's precise quadrature [1, Chapter 8]. A table of these quantities was computed.

\section{APPENDIX B. FOURIER COEFFICIENTS OF $W_{k j}^{i}$}

Recall from Lemma 3.1 that

$$
W_{k}(x, y)=\sum_{j=0}^{k} \frac{(-1)^{j}}{(2(k-j)+1) !(2 j+1) !} x^{2(k-j)+1} y^{2 j+1} \text {. }
$$

Then

$$
\begin{aligned}
W_{k 1}^{1} & =W_{k}^{1}(x, 0)=0, \\
W_{k 1}^{2} & =\left.W_{k}^{2}(x, y)\right|_{y=0}=\left.W_{k}(y, 1-x)\right|_{y=0}=W_{k}(0,1-x)=0, \\
W_{k 1}^{3} & =\left.W_{k}^{3}(x, y)\right|_{y=0}=\left.W_{k}(1-x, 1-y)\right|_{y=0}=W_{k}(1-x, 1) \\
& =\sum_{j=0}^{k} \frac{(-1)^{j}}{(2(k-j)+1) !(2 j+1) !}(1-x)^{2(k-j)+1}, \\
W_{k 1}^{4} & =\left.W_{k}^{4}(x, y)\right|_{y=0}=\left.W_{k}(1-y, x)\right|_{y=0}=W_{k}(1, x) \\
& =\sum_{j=0}^{k} \frac{(-1)^{j}}{(2(k-j)+1) !(2 j+1) !} x^{2 j+1} .
\end{aligned}
$$

Hence,

$$
\begin{aligned}
\int_{0}^{1} W_{k 1}^{3}(x) \sin m \pi x d x \\
\quad=\sum_{j=0}^{k} \frac{(-1)^{j}}{(2(k-j)+1) !(2 j+1) !} \int_{0}^{1}(1-x)^{2(k-j)+1} \sin m \pi x d x \\
\quad=\sum_{j=0}^{k} \frac{(-1)^{j}}{(2(k-j)+1) !(2 j+1) !}(-1)^{m+1} \int_{0}^{1} x^{2(k-j)+1} \sin m \pi x d x \\
\quad=\sum_{j=0}^{k} \frac{(-1)^{m+j+1}}{(2(k-j)+1) !(2 j+1) !}\left(G_{2(k-j)+1, m}(1)-G_{2(k-j)+1, m}(0)\right)
\end{aligned}
$$

and 


$$
\begin{aligned}
\int_{0}^{1} W_{k 1}^{4}(x) \sin m \pi x d x & =\sum_{j=0}^{k} \frac{(-1)^{j}}{(2(k-j)+1) !(2 j+1) !} \int_{0}^{1} x^{2 j+1} \sin m \pi x d x \\
& =\sum_{j=0}^{k} \frac{(-1)^{j}}{(2(k-j)+1) !(2 j+1) !}\left(G_{2 j+1, m}(1)-G_{2 j+1, m}(0)\right) .
\end{aligned}
$$

Similarly, the integrals $\int_{0}^{1} W_{k 2}^{i}(x) \sin m \pi x d x, \int_{0}^{1} W_{k 3}^{i}(x) \sin m \pi x d x$ and $\int_{0}^{1} W_{k 4}^{i}(x) \sin m \pi x d x$ can be expressed in terms of the functions $G_{i, m}$ evaluated at 0 and 1.

\section{BIBLIOGRAPHY}

1. O. Aberth, Precise numerical analysis, Brown, Dubuque, IA 1988.

2. A. H. Schatz and L. B. Wahlbin, Maximum norm estimates in the finite element method on plane polygonal domains. Part 1, Math. Comp. 32 (1978), 73-109.

3. F. Stenger, $A$ "Sinc-Galerkin" method of solution of boundary value problems, Math. Comp. 33 (1979), 85-109.

4. M. H. Protter and H. F. Weinberger, Maximum principles in differential equations, PrenticeHall, Englewood Cliffs, NJ, 1967.

5. John R. Rice, Ronald F. Boisvert, Solving elliptic problems using ELLPACK, Springer-Verlag, New York, 1985.

6. I. S. Gradshteyn and I. M. Ryzhik, Table of integrals, series, and products, Academic Press, New York, 1965.

7. E. Isaacson and H. B. Keller, Analysis of numerical methods, Wiley, New York 1966.

8. K. Nijima, A posteriori error bounds for piecewise linear approximate solutions of elliptic equations of monotone type, Math. Comp. 58 (1992), 549-560.

9. J. B. Rosser, Calculation of potential in a sector, Part I, MRC Technical Summary Report \#1535, May 1975.

10. Harmonic functions on regions with reentrant corners, Part I, MRC Technical Summary Report \#1796, October 1977.

Department of Mathematics, State University of New York at Buffalo, Buffalo, NEW YORK 14214

E-mail address: zshi@acsu.buffalo.edu

Department of Mathematics, State University of New York at Buffalo, Buffalo, NEW YORK 14214

E-mail address: mthbdhQubvms.cc.buffalo.edu 